\title{
Crystal structure of the new modification of tetraphenylphosphonium chloride, $\beta-\mathrm{P}\left(\mathrm{C}_{6} \mathrm{H}_{5}\right)_{4} \mathrm{Cl}$
}

\author{
H. Gruber and U. Müller \\ Universität Gh Kassel. Fachbereich Chemie/Biologie. D-34109 Kassel, Germany
}

Received February 24, 1997, CSD-No. 402866
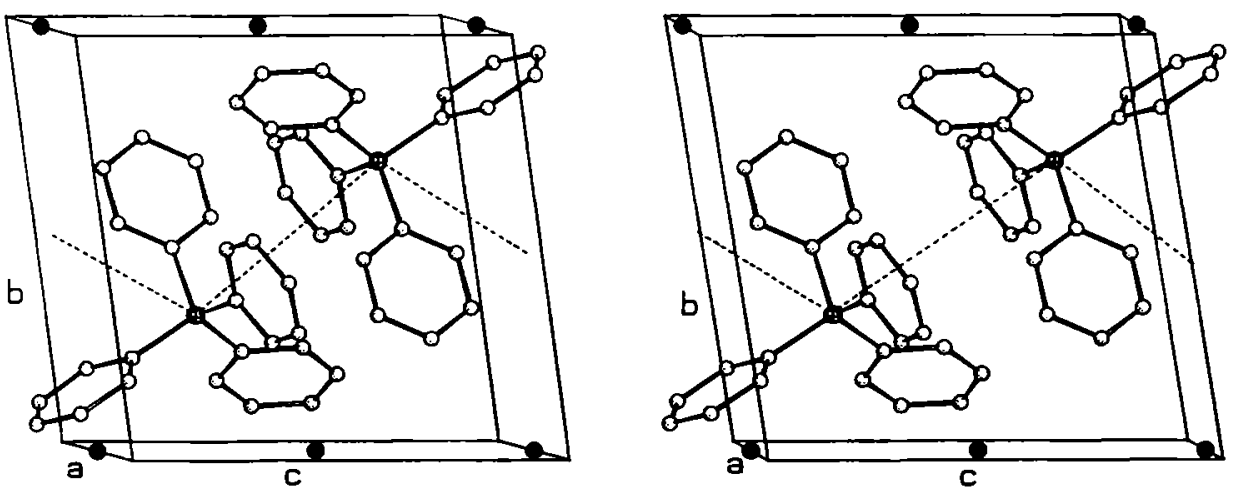

$\mathrm{C}_{24} \mathrm{H}_{20}$ ClP, triclinic, $P \overline{1}$ (No. 2), $a=9.387$ (1) $\AA, b=10.201$ (1) $\AA$, $c=11.248(1) \AA, \alpha=75.83(1)^{\circ}$, $\beta=71.42(1)^{\circ}, \gamma=78.46(1)^{\circ}, V=981.1 \AA^{3}$, $Z=2, R(F)=0.036, R_{w}\left(F^{2}\right)=0.092$.

Source of material: A solution of $1.66 \mathrm{~g} \mathrm{PPh} 4 \mathrm{Cl}$ in $70 \mathrm{ml}$ of dry acetonitrile was stirred for $15 \mathrm{~h}$ together with $0.32 \mathrm{~g} \mathrm{Na}_{2} \mathrm{~S}_{4}$ and $0.16 \mathrm{~g} \mathrm{CoCl}_{2}$, filtered and evaporated to $10 \mathrm{ml}$. $\mathrm{B}-\mathrm{PPh}_{4} \mathrm{Cl}$ crystallized at $253 \mathrm{~K}$.

In the new modification $\beta-\mathrm{PPh}_{4} \mathrm{Cl}$ the tetraphenylphosphonium ions are arranged to zigzag chains, in a similar way as in $\beta$ ( $\left.\mathrm{PPh}_{4}\right)_{2}\left[\mathrm{UCl}_{6}\right] \cdot \mathrm{CH}_{2} \mathrm{Cl}_{2}$ (see refs. 1, 2). The known monoclinic modification $\alpha-\mathrm{PPh}_{4} \mathrm{Cl}$ (see ref. 3) as well as $\mathrm{PPh} 4 \mathrm{Br}$ (see ref. 4) and $\mathrm{PPh}_{4} \mathrm{I}$ (see refs. 5, 6) crystallize with different packings.

Table 1. Parameters used for the X-ray data collection

\begin{tabular}{ll}
\hline Crystal: & colorless, compact, size $0.25 \times 0.31 \times 0.42 \mathrm{~mm}$ \\
Wavelength: & Mo $\mathrm{Ka}_{\alpha}$ radiation $(0.71069 \AA)$ \\
$\mu:$ & $2.81 \mathrm{~cm}^{-1}$ \\
Diffractometer: & Enraf-Nonius CAD4 \\
Scan mode: & $\omega$ \\
$\mathrm{T}_{\text {measurement: }}$ & $297 \mathrm{~K}$ \\
$2 \theta_{\text {max }}:$ & $42^{\circ}$ \\
$\mathrm{N}\left(h k l_{\text {unique: }}\right.$ & 1778 \\
Criterion for $I_{\mathrm{o}}:$ & $I_{0}>2 \sigma\left(I_{0}\right)$ \\
$\mathrm{N}(\text { param })_{\text {refined: }}$ & 238 \\
Programs: & SHELXS-86, SHELXL-93, SCHAKAL
\end{tabular}

Table 2. Final atomic coordinates and displacement parameters (in $\AA^{2}$ )

\begin{tabular}{|c|c|c|c|c|c|}
\hline Atom & Site & $x$ & $y$ & $z$ & $U_{\text {iso }}$ \\
\hline$H(2)$ & $2 i$ & $-0.2170(4)$ & $0.7945(4)$ & $0.3258(3)$ & 0.069 \\
\hline $\mathbf{H}(3)$ & $2 i$ & $-0.4632(5)$ & $0.7468(5)$ & $0.3944(4)$ & 0.088 \\
\hline$H(4)$ & $2 i$ & $-0.5148(5)$ & $0.5466(5)$ & $0.3713(4)$ & 0.093 \\
\hline$H(5)$ & $2 i$ & $-0.3230(5)$ & $0.3882(4)$ & $0.2876(4)$ & 0.092 \\
\hline$H(6)$ & $2 i$ & $-0.0737(4)$ & $0.4307(4)$ & $0.2228(3)$ & 0.069 \\
\hline $\mathrm{H}(8)$ & $2 i$ & $-0.0478(4)$ & $0.6858(4)$ & $0.4630(3)$ & 0.067 \\
\hline$H(9)$ & $2 i$ & $0.0186(5)$ & $0.7941(4)$ & $0.5908(4)$ & 0.084 \\
\hline$H(10)$ & $2 i$ & $0.2210(5)$ & $0.9162(4)$ & $0.5090(4)$ & 0.076 \\
\hline$H(11)$ & $2 i$ & $0.3549(4)$ & $0.9331(3)$ & $0.2971(4)$ & 0.066 \\
\hline$H(12)$ & $2 i$ & $0.2887(4)$ & $0.8298(3)$ & $0.1665(3)$ & 0.057 \\
\hline$H(14)$ & $2 i$ & $0.2211(4)$ & $0.4754(3)$ & $0.3642(3)$ & 0.059 \\
\hline$H(15)$ & $2 i$ & $0.3662(4)$ & $0.2624(4)$ & $0.3608(4)$ & 0.073 \\
\hline$H(16)$ & $2 i$ & $0.4342(4)$ & $0.1713(4)$ & $0.1795(4)$ & 0.078 \\
\hline $\mathrm{H}(17)$ & $2 i$ & $0.3645(4)$ & $0.2902(4)$ & $0.0015(4)$ & 0.082 \\
\hline$H(18)$ & $2 i$ & $0.2133(4)$ & $0.4990(4)$ & $0.0051(4)$ & 0.072 \\
\hline$H(20)$ & $2 i$ & $0.3074(4)$ & $0.7614(4)$ & $-0.0215(3)$ & 0.073 \\
\hline H(21) & $2 i$ & $0.3129(5)$ & $0.8925(4)$ & $-0.2225(3)$ & 0.079 \\
\hline $\mathrm{H}(22)$ & $2 i$ & $0.0922(5)$ & $0.9685(4)$ & $-0.2790(4)$ & 0.074 \\
\hline$H(23)$ & $2 i$ & $-0.1353(5)$ & $0.9188(4)$ & $-0.1372(4)$ & 0.079 \\
\hline$H(24)$ & $2 i$ & $-0.1446(4)$ & $0.7915(3)$ & $0.0642(3)$ & 0.065 \\
\hline
\end{tabular}

Table 3. Final atomic coordinates and displacement parameters (in $\AA^{2}$ )

\begin{tabular}{|c|c|c|c|c|c|c|c|c|c|c|}
\hline Atom & Site & $x$ & $y$ & $z$ & $U_{11}$ & $U_{22}$ & $U_{33}$ & $U_{12}$ & $U_{13}$ & $U_{23}$ \\
\hline $\mathrm{Cl}(1)$ & $1 f$ & $1 / 2$ & 0 & $1 / 2$ & $0.072(1)$ & $0.0633(9)$ & $0.0668(9)$ & $-0.0178(7)$ & $-0.0360(7)$ & $-0.0099(7)$ \\
\hline $\mathrm{Cl}(2)$ & id & $1 / 2$ & 0 & 0 & $0.099(1)$ & $0.069(1)$ & $0.084(1)$ & $-0.0355(9)$ & $0.0378(9)$ & $-0.0403(8)$ \\
\hline $\mathrm{P}$ & $2 i$ & $0.06948(9)$ & $0.66085(8)$ & $0.19876(8)$ & $0.0400(6)$ & $0.0414(5)$ & $0.0392(5)$ & $-0.0018(4)$ & $-0.0101(4)$ & $-0.0074(4)$ \\
\hline$C(1)$ & $2 i$ & $-0.1188(4)$ & $0.6177(3)$ & $0.2660(3)$ & $0.040(2)$ & $0.042(2)$ & $0.044(2)$ & $-0.006(2)$ & $-0.014(2)$ & $-0.007(2)$ \\
\hline$C(2)$ & $2 i$ & $-0.2375(4)$ & $0.7119(4)$ & $0.3182(3)$ & $0.046(3)$ & $0.056(2)$ & $0.070(3)$ & $-0.002(2)$ & $-0.016(2)$ & $-0.015(2)$ \\
\hline $\mathrm{C}(3)$ & $2 i$ & $-0.3847(5)$ & $0.6840(5)$ & $0.3585(4)$ & $0.046(3)$ & $0.089(3)$ & $0.079(3)$ & $0.000(2)$ & $-0.012(2)$ & $-0.021(2)$ \\
\hline$C(4)$ & $2 i$ & $-0.4150(5)$ & $0.5645(5)$ & $0.3456(4)$ & $0.047(3)$ & $0.107(4)$ & $0.080(3)$ & $-0.028(3)$ & $-0.009(2)$ & $-0.019(3)$ \\
\hline
\end{tabular}


Table 3. (Continued)

\begin{tabular}{|c|c|c|c|c|c|c|c|c|c|c|}
\hline Atom & Site & $x$ & $y$ & $z$ & $U_{11}$ & $U_{22}$ & $U_{33}$ & $U_{12}$ & $U_{13}$ & $U_{23}$ \\
\hline$C(5)$ & $2 i$ & $-0.3006(5)$ & $0.4700(4)$ & $0.2953(4)$ & $0.073(3)$ & $0.080(3)$ & $0.084(3)$ & $-0.036(3)$ & $-0.012(3)$ & $-0.02 !(2)$ \\
\hline$C(6)$ & $2 i$ & $-0.1515(4)$ & $0.4956(4)$ & $0.2559(3)$ & $0.054(3)$ & $0.057(2)$ & $0.060(2)$ & $-0.013(2)$ & $-0.010(2)$ & $-0.012(2)$ \\
\hline$C(7)$ & $2 i$ & $0.1129(3)$ & $0.7482(3)$ & $0.3002(3)$ & $0.040(2)$ & $0.037(2)$ & $0.038(2)$ & $-0.001(2)$ & $-0.012(2)$ & $-0.004(2)$ \\
\hline $\mathrm{C}(8)$ & $2 i$ & $0.0329(4)$ & $0.7366(4)$ & $0.4286(3)$ & $0.059(2)$ & $0.065(2)$ & $0.045(2)$ & $-0.022(2)$ & $-0.009(2)$ & $-0.011(2)$ \\
\hline$C(9)$ & $2 i$ & $0.0736(5)$ & $0.8007(4)$ & $0.5051(4)$ & $0.081(3)$ & $0.090(3)$ & $0.043(2)$ & $-0.026(3)$ & $-0.011(2)$ & $-0.018(2)$ \\
\hline$C(10)$ & $2 i$ & $0.1937(5)$ & $0.8738(4)$ & $0.4567(4)$ & $0.074(3)$ & $0.066(3)$ & $0.061(3)$ & $-0.013(2)$ & $-0.029(2)$ & $-0.017(2)$ \\
\hline$C(12)$ & $2 i$ & $0.2336(4)$ & $0.8219(3)$ & $0.2522(3)$ & $0.043(2)$ & $0.050(2)$ & $0.044(2)$ & $-0.007(2)$ & $-0.008(2)$ & $-0.005(2)$ \\
\hline$C(13)$ & $2 i$ & $0.2001(3)$ & $0.5072(3)$ & $0.1863(3)$ & $0.038(2)$ & $0.043(2)$ & $0.044(2)$ & $-0.001(2)$ & $-0.009(2)$ & $-0.010(2)$ \\
\hline$C(14)$ & $2 i$ & $0.2478(4)$ & $0.4373(3)$ & $0.2916(3)$ & $0.044(2)$ & $0.049(2)$ & $0.049(2)$ & $-0.004(2)$ & $-0.010(2)$ & $-0.007(2)$ \\
\hline$C(15)$ & $2 i$ & $0.3355(4)$ & $0.3103(4)$ & $0.2895(4)$ & $0.053(2)$ & $0.048(2)$ & $0.069(3)$ & $0.001(2)$ & $-0.019(2)$ & $0.004(2)$ \\
\hline$C(16)$ & $2 i$ & $0.3764(4)$ & $0.2567(4)$ & $0.1810(4)$ & $0.052(2)$ & $0.045(2)$ & $0.091(3)$ & $0.004(2)$ & $-0.014(2)$ & $-0.018(3)$ \\
\hline$C(17)$ & $2 i$ & $0.3334(4)$ & $0.3269(4)$ & $0.0751(4)$ & $0.064(3)$ & $0.063(3)$ & $0.081(3)$ & $0.011(2)$ & $-0.019(2)$ & $-0.037(2)$ \\
\hline$C(18)$ & $2 i$ & $0.2439(4)$ & $0.4521(4)$ & $0.0768(4)$ & $0.063(3)$ & $0.060(3)$ & $0.059(2)$ & $0.006(2)$ & $-0.024(2)$ & $-0.021(2)$ \\
\hline$C(19)$ & $2 i$ & $0.0800(4)$ & $0.7623(3)$ & $0.0422(3)$ & $0.048(2)$ & $0.041(2)$ & $0.041(2)$ & $0.000(2)$ & $-0.015(2)$ & $-0.008(2)$ \\
\hline$C(21)$ & $2 i$ & $0.2210(5)$ & $0.8711(4)$ & $-0.1644(3)$ & $0.072(3)$ & $0.071(3)$ & $0.041(2)$ & $-0.011(2)$ & $-0.002(2)$ & $-0.004(2)$ \\
\hline $\mathrm{C}(22)$ & $2 i$ & $0.0892(5)$ & $0.9168(4)$ & $-0.1979(4)$ & $0.093(3)$ & $0.047(2)$ & $0.047(2)$ & $-0.003(2)$ & $-0.029(3)$ & $-0.006(2)$ \\
\hline$C(23)$ & $2 i$ & $-0.0463(5)$ & $0.8872(4)$ & $-0.1136(4)$ & $0.072(3)$ & $0.059(3)$ & $0.069(3)$ & $-0.007(2)$ & $-0.036(2)$ & $0.006(2)$ \\
\hline$C(24)$ & $2 i$ & $-0.0517(4)$ & $0.8107(3)$ & $0.0066(3)$ & $0.057(3)$ & $0.051(2)$ & $0.053(2)$ & $-0.009(2)$ & $-0.022(2)$ & $0.001(2)$ \\
\hline
\end{tabular}

Acknowledgment. We thank the Fonds der Chemischen Industrie for support.

\section{References}

1. Müller, U.; Klingelhöfer, P.; Eicher, J.; Bohrer, R.: Die Packung in drei verschiedenen Strukturtypen für Verbindungen $\left(\mathrm{AsPh}_{4}\right)_{2}\left[\mathrm{MCl}_{5} \mathrm{X}\right]$ - $2 \mathrm{CH}_{2} \mathrm{Cl}_{2}$. Die Kristallstrukturen von $\left(\mathrm{AsPh}_{4}\right)_{2}\left[\mathrm{TiCl}_{6}\right] \cdot 2 \mathrm{CH}_{2} \mathrm{Cl}_{2}$ und $\left(\mathrm{AsPh}_{4}\right)_{2}\left[\mathrm{UCl}_{6}\right] \cdot 2 \mathrm{CH}_{2} \mathrm{Cl}_{2}$. Z. Kristallogr. 168 (1984) 121-131.

2. Conradi, E.; Bohrer, R.; Weber, R.; Müller, U.: Die Kristallstrukturen von (PPh4) $\left[\mathrm{RuCl}_{6}\right] \cdot 2 \mathrm{CH}_{2} \mathrm{Cl}_{2}$, (AsPh4) $\left[\mathrm{OsCl}_{6}\right] \cdot 2 \mathrm{CH}_{2} \mathrm{Cl}_{2}$ und $\beta$ $\left(\mathrm{PPh}_{4}\right)_{2}\left[\mathrm{UCl}_{6}\right] \cdot 2 \mathrm{CH}_{2} \mathrm{Cl}_{2}$. Z. Kristallogr. 181 (1987) 187-198.

3. Richardson J. F.; Ball, J. M.; Boorman, P. M.: Structure of tetraphenylphosphonium chloride. Acta Crystallogr. C42 (1986) 1271-1272.
4. Schweizer, E. E.; Baldacchini, C. J.; Rheingold, A. L.: Tetraphenylphosphonium chloride monohydrate, tetraphenylphosphonium bromide and tetraphenylphosphonium iodide. Acta Crystallogr. C45 (1989) 1236-1239.

5. Khotsyanova, T. L.; Struchkov, Ya. T.: Structure of tetraphenylphosphonium iodide. Kristallografiya 1 (1967) 669.

6. Harms, K.: XCAD4, Programm zur Datenreduktion von CAD4-Meßdaten. University of Marburg, Germany 1987.

7. Sheldrick, G. M.: SHELXS-86, Program for the solution of crystal structures. University of Göttingen, Germany 1986.

8. Sheldrick, G. M.: SHELXL-93, Program for crystal structure determination. University of Göttingen, Germany 1993.

9. Keller, L.: SCHAKAL-92, Program for the graphic representation of crystal models. University of Freiburg, Germany 1992. 